\title{
Genetic risk for schizophrenia and developmental delay is associated with shape and microstructure of midline white- matter structures
}

\author{
Mark Drakesmith (1), ${ }^{1,2}$ Greg D. Parker ${ }^{1,2,3}$, Jacqueline Smith ${ }^{2,4}$, Stefanie C. Linden ${ }^{2,4}$, Elliott Rees ${ }^{2,4}$, Nigel Williams ${ }^{2,4}$,
} Michael J. Owen ${ }^{2,4}$, Marianne van den Bree ${ }^{2,4}$, Jeremy Hall ${ }^{2,4}$, Derek K. Jones ${ }^{1,2,5}$ and David E. J. Linden ${ }^{1,2,46}$

\begin{abstract}
Genomic copy number variants (CNVs) are amongst the most highly penetrant genetic risk factors for neuropsychiatric disorders. The scarcity of carriers of individual CNVs and their phenotypical heterogeneity limits investigations of the associated neural mechanisms and endophenotypes. We applied a novel design based on CNV penetrance for schizophrenia (Sz) and developmental delay (DD) that allows us to identify structural sequelae that are most relevant to neuropsychiatric disorders. Our focus on brain structural abnormalities was based on the hypothesis that convergent mechanisms contributing to neurodevelopmental disorders would likely manifest in the macro- and microstructure of white matter and cortical and subcortical grey matter. Twenty one adult participants carrying neuropsychiatric risk CNVs (including those located at 22q11.2, 15q11.2, 1q21.1, 16p11.2 and 17q12) and 15 age- and gender-matched controls underwent T1-weighted structural, diffusion and relaxometry MRI. The macro- and microstructural properties of the cingulum bundles were associated with penetrance for both developmental delay and schizophrenia, in particular curvature along the anterior-posterior axis (Sz: $p_{\text {corr }}=0.026$; DD: $p_{\text {corr }}=0.035$ ) and intracellular volume fraction (Sz: $p_{\text {corr }}=0.019$; DD: $p_{\text {corr }}=0.064$ ). Further principal component analysis showed alterations in the interrelationships between the volumes of several midline white-matter structures (Sz: $p_{\text {corr }}=0.055$; $\left.\mathrm{DD}: p_{\text {corr }}=0.027\right)$. In particular, the ratio of volumes in the splenium and body of the corpus callosum was significantly associated with both penetrance scores ( $\mathrm{Sz}: p=0.037 ; \mathrm{DD} ; p=0.006$ ). Our results are consistent with the notion that a significant alteration in developmental trajectories of midline white-matter structures constitutes a common neurodevelopmental aberration contributing to risk for schizophrenia and intellectual disability.
\end{abstract}

\section{Introduction}

Genetic variants are associated with neurodevelopmental disorders across conventional diagnostic classifications. This is evidenced both by common variants with low penetrance and by rarer but highly penetrant copy

\footnotetext{
Correspondence: Mark Drakesmith (drakesmithm@cardiff.ac.uk) ${ }^{1}$ Cardiff University Brain Research Imaging Centre (CUBRIC), School of Psychology, Cardiff University, Maindy Road, Cardiff CF24 4HQ, United Kingdom

${ }^{2}$ Neuroscience and Mental Health Research Institute (NMHRI), Cardiff University, Maindy Road, Cardiff CF24 4HQ, United Kingdom Full list of author information is available at the end of the article.
}

number variants $(\mathrm{CNVs})^{1,2}$. The mechanisms through which these genetic variants affect brain and behaviour are poorly understood, but genetic imaging studies in people selected for polygenic risk ${ }^{3}$ or $\mathrm{CNV}$ carriers ${ }^{4}$ can elucidate changes in brain development, structure and function that are not confounded by secondary disease effects. Because of their high penetrance, CNVs are particularly suited to translational studies elucidating disease mechanisms. However, rarity of CNVs makes it difficult to investigate their biological effects in humans. For most of the pathogenic $\mathrm{CNVs}$ identified for schizophrenia and

\section{(c) The Author(s) 2019}

(c) (i) Open Access This article is licensed under a Creative Commons Attribution 4.0 International License, which permits use, sharing, adaptation, distribution and reproduction c. in any medium or format, as long as you give appropriate credit to the original author(s) and the source, provide a link to the Creative Commons license, and indicate if changes were made. The images or other third party material in this article are included in the article's Creative Commons license, unless indicated otherwise in a credit line to the material. If material is not included in the article's Creative Commons license and your intended use is not permitted by statutory regulation or exceeds the permitted use, you will need to obtain permission directly from the copyright holder. To view a copy of this license, visit http://creativecommons.org/licenses/by/4.0/. 
Table 1 Summary of neuroimaging findings in targeted CNVs

\begin{tabular}{|c|c|c|}
\hline \multirow[t]{2}{*}{ CNV (hg19) } & \multicolumn{2}{|l|}{ Neuroimaging finding } \\
\hline & Morphological & Microstructural \\
\hline $\begin{array}{l}\text { 15q11.2 BP1-2 deletion } \\
\text { (chr15:22,805,313-23,094,530) }\end{array}$ & Decreased volume in fusiform gyrus ${ }^{9}$ & None \\
\hline $\begin{array}{l}\text { 15q13.3 BP4-5 duplication } \\
\text { (chr15:31,080,645-32,462,776) }\end{array}$ & Abnormal bilateral hippocampal structure ${ }^{52}$ & None \\
\hline $\begin{array}{l}\text { 15q13.3 BP4-5 deletion } \\
\text { (chr15:31,080,645-32,462,776) }\end{array}$ & Pachygyria and subcortical band heterotopia ${ }^{53}$ & None \\
\hline $\begin{array}{l}\text { 16p11.2 distal duplication } \\
\text { (chr16:28,823,196-29,046,783) }\end{array}$ & $\begin{array}{l}\text { cortical atrophy, thinning of corpus callosum, ventricular } \\
\text { enlargement }{ }^{54} \text {. }\end{array}$ & None \\
\hline $16 p 11.2$ deletion chr16:29,650,840-30,200,773 & $\begin{array}{l}\text { Increased brain size. Increased cortical grey matter }{ }^{55} \\
\text { Medio-dorsal thalamus, insula, ventral striatum, orbito- } \\
\text { frontal cortex and fronto-striatal white matter }{ }^{56,57}\end{array}$ & $\begin{array}{l}\text { Changes in fractional anisotropy and mean diffusivity in } \\
\text { reward and language pathways; striatum, middle and } \\
\text { superior temporal gyrus }{ }^{56} \text {. increased axial diffusivity in } \\
\text { many major white-matter tracts, including the anterior } \\
\text { corpus callosum, internal and external capsules. Decreases } \\
\text { in fibre orientation dispersion }\end{array}$ \\
\hline 17q12 duplication (chr17:34,815,904-36,217,432) & Brainstem atrophy and hippocampal asymmetry ${ }^{59}$ & None \\
\hline 1q21.1 deletion (chr1:146,527,987-147,394,444) & No changes found ${ }^{60}$ & None \\
\hline 1q21.1 duplication (chr1:146,527,987-147,394,444) & Reduced corpus callosum volume, enlarged ventricles ${ }^{60}$. & None \\
\hline 22q11.2 deletion (chr22:19,037,332-21,466,726) & $\begin{array}{l}\text { Whole-brain volumetric reductions, particularly in midline } \\
\text { regions }{ }^{61-63} \text {. Increased cortical thickness } 64-66 \\
\text { Rostrocaudal gradient of volume reduction?. More } \\
\text { comprehensive reviews are found in } \mathrm{i}^{5-8} \text {. }\end{array}$ & $\begin{array}{l}\text { Differences in fractional anisotropy and diffusivity } \\
\text { parameters, e.g. lower fractional anisotropy in cingulum } \\
\text { bundle and lower mean diffusivity in inferior longitudinal } \\
\text { fasiculus }{ }^{67} \text { increase in fractional anisotropy in left inferior } \\
\text { fronto-occipital fasiculus, and a decrease in RD in right } \\
\text { IFOF; increase in right hemisphere FA and a decrease in } \\
\text { right RD in right cingulum; increase FA decrease RD within } \\
\text { the right thalamo-frontal tract; decrease radial diffusivity in } \\
\text { right inferior longitudinal fasiculus }{ }^{65} \text {. }\end{array}$ \\
\hline 22q11.2 duplication (chr22:19,037,332-21,466,726) & $\begin{array}{l}\text { Greater overall grey and whit matter volumes and cortical } \\
\text { surface area. Reduced cortical thickness. larger right } \\
\text { hippocampus smaller caudate and corpus callosum } \\
\text { volume (opposite findings compared to } 22 \text { q } 11.2 \text { del) }{ }^{65}\end{array}$ & None \\
\hline 3q29 deletion (chr3:195,720,167-197,354,826) & None & None \\
\hline NRXN1 deletion (chr2:50145643-51259674) & $\begin{array}{l}\text { General lack of findings } 68 \text { except for one case of } \\
\text { hippocampal atrophy }\end{array}$ & None \\
\hline
\end{tabular}

Literature found using search terms " $<$ CNV > imaging" and " $<$ CNV > MRI". Findings based on single case studies or non-quantitative case series are italicised

developmental delay, there is no information about neuroimaging correlates beyond small case series. Most neuroimaging studies of these CNVs have assessed effects of the 22q11.2 deletion (reviewed in $^{5-8}$ ). Neuroimaging studies in carriers of other CNVs have revealed highly heterogeneous changes in brain structure across CNVs (Table 1).

Most findings relate to brain morphology (measured using regional volumes, cortical thickness, surface area etc.), with few studies reporting microstructural difference, usually measured with diffusion tensor metrics such as fractional anisotropy (FA) and mean diffusivity (MD). Although some of these findings corroborate those for non-CNV neuropsychiatric patients (e.g. structural correlates in $15 \mathrm{q} 11.2$ deletion carriers are similar to those found in dyslexia ${ }^{9}$ ), in most cases it is difficult to corroborate findings in $\mathrm{CNV}$ patients with particular clinical features.

Our survey of the neuroimaging literature in $\mathrm{CNV}$ carriers (Table 1) attests to the difficulty of collecting substantial quantitative data and drawing consistent conclusions about morphological and microstructural brain alterations in carriers from the literature, particularly of the rarer CNVs. We therefore conducted an analysis across a cohort of carriers of different CNVs, looking for features that correlate with the propensity of these CNVs to contribute to neuropsychiatric illness. We adopt a novel approach to characterising brain features in high-risk CNV carriers using penetrance scores previously calculated from a large cohort of neuropsychiatric CNV patients ${ }^{10}$. This approach allows us to take account of the degree of pathogenicity of a genetic variant, testing the hypothesis that clinically more penetrant variants will also be associated with more salient neurodevelopmental changes as detected on neuroimaging. This method has the advantage over single-CNV studies that it enables the detection of convergent pathways common to several genetic variants, which are putatively most directly related to the pathophysiology of the associated diseases.

We explore structural brain phenotypes derived from neuroimaging data. These relate to macroscopic structure of cortex, including volume, surface area and cortical thickness, and the size of subcortical regions using T1weighted structural MRI. We also quantify indices of white-matter microstructure and morphology using diffusion MRI. We quantify tract volume and tract shape 
using a novel approach that extracts principal modes of feature variation of streamline shape ${ }^{11}$. We also quantify various microstructural parameters within the principal white-matter pathways using metrics derived from diffusion tensor imaging (DTI), measures of axon density and dispersion using the neurite orientation dispersion and density imaging (NODDI) ${ }^{12}$ method and T1 relaxometry which provides a putative index of myelination ${ }^{13}$.

In addition to examining each of these features separately, we also use principal component analysis (PCA) to identify any components across all these neuroanatomical features that show a strong effect of disease penetrance. The advantage of this approach is that it can highlight any prevalent components across correlated features that are not necessarily apparent when examining individual features separately.

\section{Methods}

\section{Participants}

The study was approved by the South Wales Research Ethics Committee and the Cardiff University School of Psychology Ethics Committee. All participants provided written informed consent.

MRI data were obtained from $21 \mathrm{CNV}$ carriers and 15 controls. CNVs (Table 2) were targeted for their high association with the development of schizophrenia and developmental disorder. Patients were recruited from NHS genetics clinics within the UK and through information disseminated by relevant support groups to their members. Summary information on clinical features and intelligence for this cohort are provided in the Supplementary Information (SI, section 1). Exclusion criteria included any contraindication to MRI.

Controls were recruited via a local panel of genotyped volunteers. Control participants were chosen to match the age and gender of the $\mathrm{CNV}$ patients where possible. Criteria for inclusion were having no history of neurological or psychiatric disorders in addition to the general screening for MRI contraindications and exclusion of any of the pathogenic CNVs.

\section{Genotyping}

To confirm CNV status, all patients and controls were genotyped using the Illumina HumanCoreExome whole genome SNP array that contains an additional 27,000 genetic variants at loci that had been previously implicated in neurological and psychiatric disease, which included CNVs. After processing the raw intensity data using Illumina Genome Studio software (version 2011.1), $\log \mathrm{R}$ ratios and B allele frequencies were used to call CNVs using PennCNV (version 1.0.3) ${ }^{14}$. CNV coordinates are according to genome version hg19. CNVs were called if they spanned at least 10 informative SNPs and were joined if the distance between them was less than $50 \%$ of their combined length. CNVs were excluded if they were less than $10 \mathrm{~kb}$ in size, overlapped low copy repeats by more than $50 \%$ of their length, or had a

Table 2 Demographic data for CNV and control participants

\begin{tabular}{|c|c|c|c|c|c|c|c|}
\hline \multirow[t]{2}{*}{ CNV (hg19) } & \multicolumn{2}{|c|}{ Age (years) } & \multicolumn{2}{|c|}{ Gender } & \multirow[t]{2}{*}{$N$} & \multicolumn{2}{|c|}{ Penetrance } \\
\hline & Mean & s.d. & M & $\mathbf{F}$ & & Sz & DD \\
\hline All CNVs & 37.4 & 11.7 & 14 & 7 & 21 & 5.4 & 36.7 \\
\hline 15q11.2 BP1-2 deletion (chr15:22,805,313-23,094,530) & 48.4 & 2.3 & 1 & 1 & 2 & 2 & 11 \\
\hline 15q13.3 BP4-5 deletion (chr15:31,080,645-32,462,776) & 30.0 & 4.5 & 2 & 0 & 2 & 4.7 & 35 \\
\hline 15q13.3 BP4-5 duplication (chr15:31,080,645-32,462,776) & 41.7 & - & 1 & 0 & 1 & 1.8 & 8 \\
\hline 16p11.2 distal duplication (chr16:28,823,196-29,046,783) & 40.3 & - & 0 & 1 & 1 & 0.7 & 5.3 \\
\hline 16p11.2 deletion chr16:29,650,840-30,200,773 & 43.0 & - & 1 & 0 & 1 & 0.5 & 31 \\
\hline 17q12 duplication (chr17:34,815,904-36,217,432) & 47.1 & - & 0 & 1 & 1 & 1.7 & 17 \\
\hline 1q21.1 deletion (chr1:146,527,987-147,394,444) & 35.0 & 15.0 & 4 & 0 & 4 & 5.2 & 35 \\
\hline 1q21.1 duplication (chr1:146,527,987-147,394,444) & 39.5 & - & 0 & 1 & 1 & 2.9 & 18 \\
\hline 22q11.2 deletion (chr22:19,037,332-21,466,726) & 31.2 & 17.0 & 2 & 2 & 4 & 12 & 88 \\
\hline 22q11.2 duplication (chr22:19,037,332-21,466,726) & 44.9 & 4.8 & 1 & 1 & 2 & 0 & 14 \\
\hline 3q29 deletion (chr3:195,720,167-197,354,826) & 19.9 & - & 1 & 0 & 1 & 18 & 53 \\
\hline NRXN1 deletion (chr2:50145643-51259674) & 43.6 & - & 1 & 0 & 1 & 6.4 & 26 \\
\hline Control & 39.6 & 11.3 & 6 & 9 & 15 & 0 & 0 \\
\hline
\end{tabular}


probe density of less than 1 probe $/ 20 \mathrm{~kb}$. The Log $\mathrm{R}$ ratio and $\mathrm{B}$ allele frequency plots at each of the genomic regions of interest (chr1:146,527,987-147,394,444, chr2:50145643-512 59674, chr3:195,720,167-197,354,826, chr15:22,805,313-23, 094,530, chr15:31,080,645-32,462,776, chr16:28,823,196-29, 046,783, chr16:29,650,840-30,200,773, chr17:29,107,4 91-30,265,075, chr22:19,037,332-21,466,726) were also manually inspected in order to confirm the presence of the CNV.

\section{MRI acquisition}

The collection and analysis of all MRI measures used in this study are summarised in Fig. 1. All MRI data were acquired on a $3 \mathrm{~T}$ General Electric HDx MRI system (GE Medical Systems, Milwaukee, WI) using an eight-channel receive-only head RF coil.

\section{Structural}

T1-weighted structural images were acquired with a 3D fast spoiled gradient echo (FSPGR) sequence (TR = $7.8 \mathrm{~ms}, \mathrm{TE}=3.0 \mathrm{~ms}$, voxel size $=1 \mathrm{~mm}^{3}$ isomorphic).

\section{Diffusion}

A cardiac-gated diffusion-weighted spin-echo echo-planar imaging sequence was used to acquire high angular resolution diffusion-weighted images (HARDI ${ }^{15}$. Sixty gradient orientations at $b=2000 \mathrm{~s} / \mathrm{mm}^{2}$, and 30 directions at $b=$ $1200 \mathrm{~s} / \mathrm{mm}^{2}$, and 6 unweighted $\left(b=0 \mathrm{~s} / \mathrm{mm}^{2}\right)$ images were acquired with the following parameters: $\mathrm{TE}=87 \mathrm{~ms}$, 60 slices, slice thickness $=2.4 \mathrm{~mm}$, FoV $=230 \times 230 \mathrm{~mm}$, Acquisition matrix $=96 \times 96$, resulting in data acquired with a $2.4 \times 2.4 \times 2.4 \mathrm{~mm}$ isotropic resolution. This was followed by zero-filling to a $128 \times 128$, in-plane matrix for the fast Fourier transform. The final image resolution was therefore $1.8 \times 1.8 \times 2.4 \mathrm{~mm}$.

\section{Relaxometry}

A series of spoiled gradient echo (SPGR) images was acquired with 8 flip angles plus an additional inversionrecovery (IR) SPGR image. All images had TE $=2.11 \mathrm{~ms}$ and $T R=4.7 \mathrm{~ms}$. SPGR images were acquired with flip angles of $3^{\circ}, 4^{\circ}, 5^{\circ}, 6^{\circ}, 7^{\circ}, 9^{\circ}, 13^{\circ}$ and $18^{\circ}$. For the IR-SPGR acquisition, Inversion time $=450 \mathrm{~ms}$ and flip angle $=5^{\circ}$.

\section{Grey-matter morphometry}

Cortical reconstruction and volumetric segmentation were obtained from the T1-weighted structural images using Freesurfer (http://surfer.nmr.mgh.harvard.edu/) ${ }^{16}$. The technical details of these procedures are described in prior publications ${ }^{17-23}$. Grey matter was registered and parcelated to the Desikan-Killiany Atlas $^{24}$ (40 regions) and measures of volume, surface area, thickness and curvature were generated.

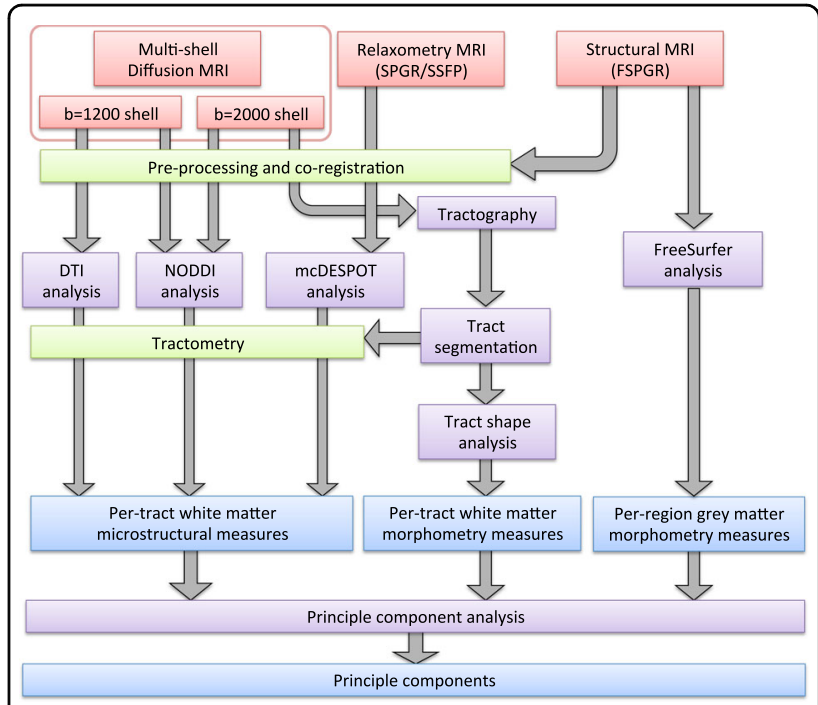

Fig. 1 Flowchart of MRI data processing pipeline. Red boxes show MRI acquisition steps. Green boxes show image registration steps. Purple boxes show main data processing steps. Blue boxes represent final derived imaging variables

\section{Relaxometry pre-processing and analysis}

Relaxometry data were pre-processed using FSL v5.0 ${ }^{25}$. All SPGR/IR-SPGR images were coregistered to each other using a rigid affine transform and skull-stripped ${ }^{26}$. Relaxation rate $(\mathrm{R} 1=1 / \mathrm{T} 1)$ maps were derived using Driven Equilibrium Single Pulse Observation of T1 with High-Speed Incorporation of RF Field Inhomogeneities (DESPOT1-HIFI $^{13}$, which incorporates correction for B1 field inhomogeneities with in-house code. A synthetic T1weighted image was computed from the quantitative T1 map with contrast matching that of the FSPGR image. This was used as a reference for transforming the R1 (maps, which were then warped to the T1-weighted space.

\section{Diffusion MRI pre-processing}

HARDI data were pre-processed in ExploreDTI v4.8.3 $3^{27}$. Data were corrected for motion, eddy currents and field inhomogeneities prior to tractography. Motion artefacts and eddy current distortions were corrected with B-matrix rotation using the approach of ref. ${ }^{28}$. A comparison of subject motion between the two groups is shown in SI, section 2.

Field inhomogeneities were corrected using the approach of ${ }^{29}$. DWIs were non-linearly warped to the T1weighted image using the fractional anisotropy map from the DWIs as a reference. Warps were computed using Elasti $^{30}$ using normalised mutual information cost function and constraining deformations to the phase-encoding direction. The corrected DWIs are therefore in the same (undistorted) space as the T1-weighted structural images. 


\section{DTI analysis}

DTI fitting was performed using ExploreDTI v4.8.3. The corrected HARDI data from the $b=1200 \mathrm{~s} / \mathrm{mm}^{2}$ shell were fitted to the diffusion tensor (DT) and corrected for CSF-partial volume effects ${ }^{31}$ was applied to the DTs. The $b=1200 \mathrm{~s} / \mathrm{mm}^{2}$ shell was used as this is the domain in which the DT representation applies. The fractional anisotropy (FA), mean (MD), axial (AD) and radial (RD) diffusivities then computed from the DT. Intra-scan head motion was quantified and assessed for potential impact on subsequent statistics (see SI, section 2).

\section{NODDI (neurite orientation dispersion and density imaging) analysis}

NODDI $^{12}$ was performed using both the NODDI toolbox (https://www.nitrc.org/projects/noddi_toolbox/) the $b=1200 \mathrm{~s} / \mathrm{mm}^{2}$ and $b=2000 \mathrm{~s} / \mathrm{mm}^{2}$ diffusion shells using the NODDI toolbox v0.9. NODDI yields three parameters that describe the microstructure of the tissue in each voxel, intracellular volume fraction (ICVF), isotropic fraction (ISOF) and orientation dispersion index (ODI).

\section{Tractography}

Whole-brain tractography was performed using the damped Richardson-Lucy algorithm using in-house MATLAB $\operatorname{code}^{32}$. This is a modified spherical deconvolution method which is more robust to spurious peaks in the fibre orientation distribution (FOD) than standard spherical deconvolution methods ${ }^{33}$. RESDORE ${ }^{34}$ was also applied to remove corrupted voxels from the FOD calculation. The tractography algorithm used is that of Basser et al. ${ }^{35}$, which uses a uniform step size. Seed points were arranged in a $3 \times 3 \times 3 \mathrm{~mm}$ grid in white matter, step size $=1 \mathrm{~mm}$, angle threshold $=45^{\circ}$, length threshold $=$ $20-500 \mathrm{~mm}$, FOD threshold $=0.05, \beta=1.77, \lambda=0.0019$, $\eta=0.04$, number of iterations $=200$ (See ref. ${ }^{32}$ for full details of these parameters).

Additional anatomical constraints were introduced to ensure minimal contamination from spurious streamline trajectories through grey matter. A segmentation of the T1-weighted images was performed using FSL-FAST and was used to apply a mask to the streamlines, such that streamlines were forced to terminate when they entered grey matter. There were no explicit masking of CSF, however, the termination criteria used for the dRL algorithm, which is based on the amplitude of the FOD peak, ensures that no streamlines enter isotropic regions such as CSF.

\section{Tract segmentation and shape analysis}

Tract shape analyses was performed to quantify the shapes of white-matter pathways using in-house
MATLAB code $^{11}$. Subject whole-brain tractography results were affinely (registered to a standard MNI template (preserving shape but eliminating variance in position/orientation). Streamlines were then re-parameterised to 30 knot-points (spline interpolation), translated to the origin and reduced to a feature vector through coordinate concatenation. PCA was then applied to determine principal modes of feature vector variation and, by extension, variation of streamline shape. Following decomposition of the feature vectors onto the first seven PCA eigenvectors (representing a set of streamline shape basis functions encapsulating $\sim 95 \%$ of observed shape variation), the resultant weight vectors were clustered (k-means, $k=$ 800) and, for each subject, histograms of streamline cluster membership recorded.

Streamlines were segmented into the following 19 tract bundles specific pathways, based on the descriptors computed from previously generated statistical models: bilateral arcuate, uncinate, inferior longitudinal, superior longitudinal, fronto-occipital fasciculi, cingulum (dorsal and parahippocampal parts), fornix (left and right branches) and corpus callosum (splenium, body and genu). This yielded a total of 194 shape descriptors. In addition to shape, volume of each bundle was also computed by counting voxels traversed by streamlines in each bundle and normalising to voxel volume. All nine microstructural measurements (from DTI, relaxometry and NODDI) were registered to streamline points in each bundle and the median value taken for each bundle. This yields a total of 190 (19 bundles $\times 10$ variables) microstructural variables.

\section{CNV penetrance scores}

To assess the contribution of genetic loading for each $\mathrm{CNV}$ for psychopathology, and to accommodate the small sample size of each individual CNV within the cohort, penetrance cores were used for statistical analysis. The $\mathrm{CNV}$ penetrance scores used in the current study correspond to the probability of manifesting a given phenotype in carriers of that CNV. Estimates of CNV penetrance for the development of schizophrenia and ID/DD/ASD were obtained from Kirov et al. ${ }^{10}$. These authors used CNV data from large cohorts of patients diagnosed with schizophrenia, ID/DD/ASD and non-psychiatric controls, to estimate the rate of specific neuropsychiatric CNVs among these disease and non-disease populations, and then estimated $\mathrm{CNV}$ penetrance as the probability of carrying a specific $\mathrm{CNV}$ given disease status (i.e. rate of the CNV in schizophrenia or ID/DD/ASD), divided by the probability of carrying the $\mathrm{CNV}$ in the general population (which includes both case and control populations). Full details on how CNV penetrance scores were calculated can be found in ref. ${ }^{10}$. 


\section{Statistical analysis}

To assess the contribution of genetic loading for each $\mathrm{CNV}$ for psychopathology, and to accommodate the small sample size of each individual CNV within the cohort, each participant was assigned a penetrance score for schizophrenia and developmental delay, as previously computed in Kirov et al. ${ }^{10}$.

A general linear model was applied to identify relationships between the penetrance scores and the imaging variable of interest. Age and gender were included as covariates. Furthermore, for volumetric measures (including tract volume), total brain volume was treated as a covariate and intra-scan head motion was included as a covariate for all diffusion-derived measures. The correlation between the two penetrance scores was also computed.

Multiple comparisons were corrected with permutation testing (5000 iterations) correcting across all observations within the same imaging variable. Permutation correction will also control for any distributions that are nonGaussian. In the case of microstructural measurements, this is corrected across the 19 fibre bundles for each microstructural variable. For the shape descriptors, the correction is applied across the 194 shape descriptors. For macrostructural morphometry measures, correction was applied across the 40 atlas regions for each macrostructural measurement. To verify effects are due to penetrance and not simply for the presence of CNVs, equivalent analysis was performed using only a binary classification of $\mathrm{CNV}$ carriers vs controls. To assess contributions of individual CNVs which may be driving any significant associates identified, the statistical analysis was repeated with individual $\mathrm{CNVs}$ omitted and the change in effect size quantified (see SI, section 3).

To identify multivariate features in the data, a principal component analysis (PCA) of all imaging variables was performed. The components were subject to the same statistical analysis as the variables.

\section{Results}

\section{Relation between penetrance scores}

There was a significant correlation between the penetrance scores $P_{\mathrm{Sz}}$ and $P_{\mathrm{DD}}\left(\rho=0.77, p=7.7 \times 10^{-9}\right)$.

\section{White-matter morphology}

Shape analysis revealed significant alteration of one component of the left cingulum bundle that was significantly correlated with both $P_{\mathrm{Sz}}(t=4.195, p=2.11 \times$ $\left.10^{-4}, p_{\text {corr }}=0.026\right)$ and $P_{\mathrm{DD}}\left(t=4.06, p=3.1 \times 10^{-5}\right.$, $\left.p_{\text {corr }}=0.035\right)$. This component reflects the curvature of the dorsal cingulum along the anterior-posterior axis (Fig. 2) with higher penetrance associated with greater curvature of the dorsal cingulum. The same effect was seen in the corresponding component of the right dorsal cingulum with $P_{\mathrm{DD}}\left(t=-5.60, p=1.81 \times 10^{-6}, p_{\mathrm{corr}}=\right.$ $\left.8.00 \times 10^{-4}\right)$. A strong effect was also observed for $\mathrm{P}_{\mathrm{Sz}}$, but this did not survive permutation correction $(t=-3.601$, $\left.p=0.001, p_{\text {corr }}=0.11\right)$. In the binary comparison of CNV carriers vs controls, these effects were non-significant (left cingulum: $t=1.41, p=0.17$; right cingulum: $t=-1.94$, $p=0.062)$. Note that the sign of the descriptor from the analysis is arbitrary, so although the effect was in the opposite direction in the right compared to the left, the modes of variation also are also reversed between left and right. Therefore both left and right cingulum bundles are showing the same geometric variation-greater curvature associated with higher penetrance. No other tract shape descriptors showed any significant effect although there were marginal effects of $P_{\mathrm{Sz}}$ on shape in the right parahippocampal cingulum and cortico-spinal tract (see SI, section 4 for full results).

Tract volumes show significant effects of $\mathrm{P}_{\mathrm{Sz}}$ on the right cortico-spinal tract $\left(t=-3.163, p=0.0035, p_{\text {corr }}=\right.$ 0.030 ) and a marginal effect on the right uncinate fasiculus $\left(t=-2.881, p=0.0072, p_{\text {corr }}=0.057\right) . P_{\mathrm{DD}}$ was associated with lower volumes of the right cingulum $(t=$ $\left.-3.698, p=8.4 \times 10^{-4}, \quad p_{\text {corr }}=0.0094\right)$ and the right uncinate fasiculus $\left(t=-3.617, p=0.0010, p_{\text {corr }}=0.011\right)$. Right cortico-spinal tract $(t=-2.605, p=0.014)$ and right uncinate fasiculus $(t=-2.093, p=0.014)$ also show some effects using the binary comparison, indicating significant group differences.

\section{White-matter microstructure}

There were significant correlations with both penetrance scores with ICVF in the left cingulum (Sz: $t=-3.258, \quad p=0.003, \quad p_{\text {corr }}=0.019 ; \quad$ DD: $t=-2.644$, $\left.p=0.013, p_{\text {corr }}=0.064\right)$. There was also a significant association between $\mathrm{P}_{\mathrm{Sz}}$ and FA (Sz: $t=-3.290$, $\left.p=0.002, p_{\text {corr }}=0.017\right)$. No significant associated of diffusivity measures were observed, but the general pattern is that penetrance scores show a negative relationship with $\mathrm{FA}$ and $\mathrm{AD}$ and positive relationship with $\mathrm{MD}$ and $\mathrm{RD}$. These findings are summarised in Fig. 3. Other findings relating to microstructural metrics were a significant positive relationship between $P_{\mathrm{Sz}}$ and ODI in left and right inferior fronto-occipital fasciculi (left: $t=3.757, p=7.1 \times$ $10^{-4}, p_{\text {corr }}=0.0072$; right: $t=4.034, p=3.3 \times 10^{-4}, p_{\text {corr }}$ $=0.0042$ ). No effects were observed for R1 (see SI section 4 for full results).

\section{Other structural measures}

Comparison of whole-brain volume showed no significant effects (Sz: $t=0.392, p=0.697$; DD: $t=0.722$, $p=0.475)$. No other grey-matter morphometric or in gross brain morphometric measures showed any significant association (see SI, section 4 for full results). 


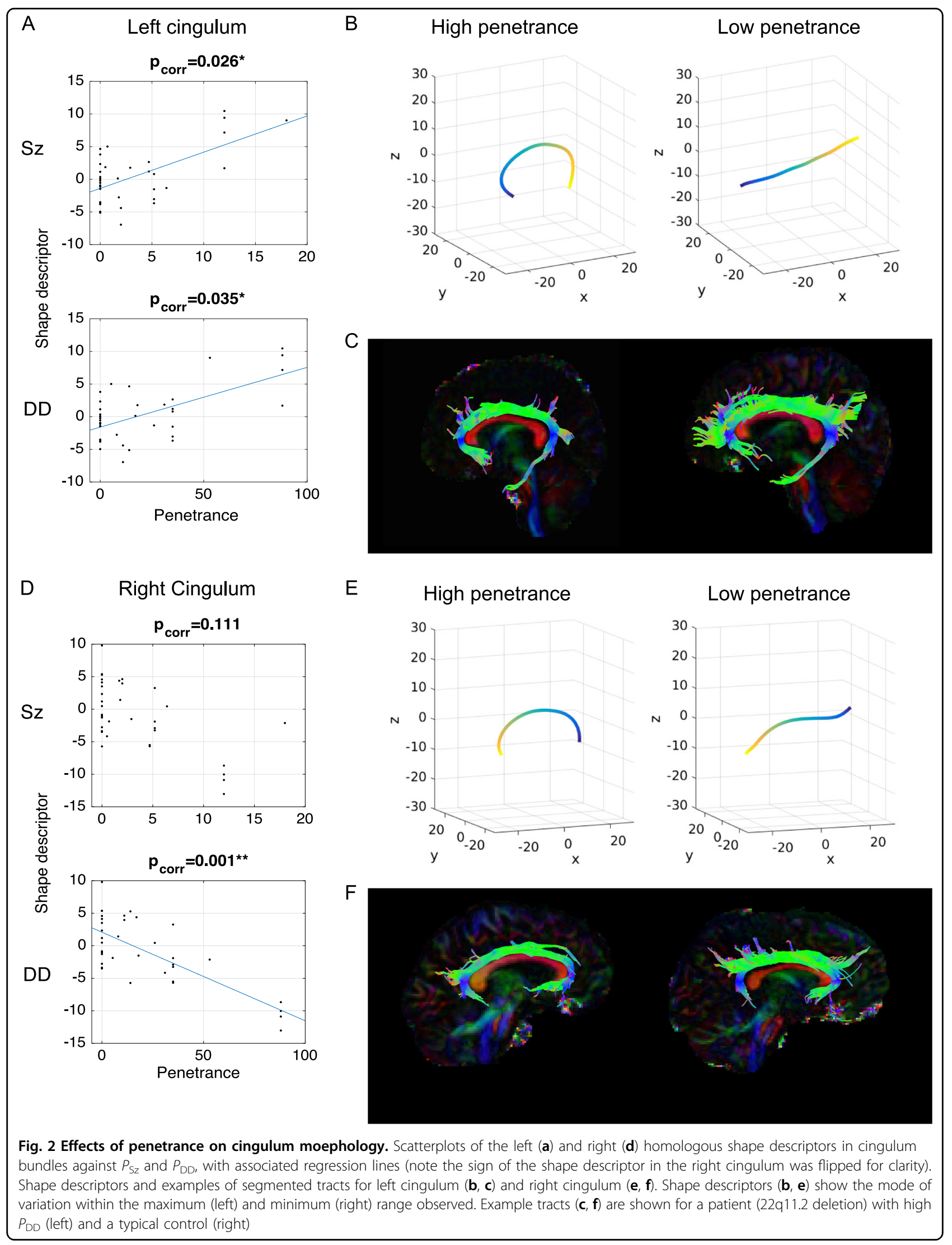




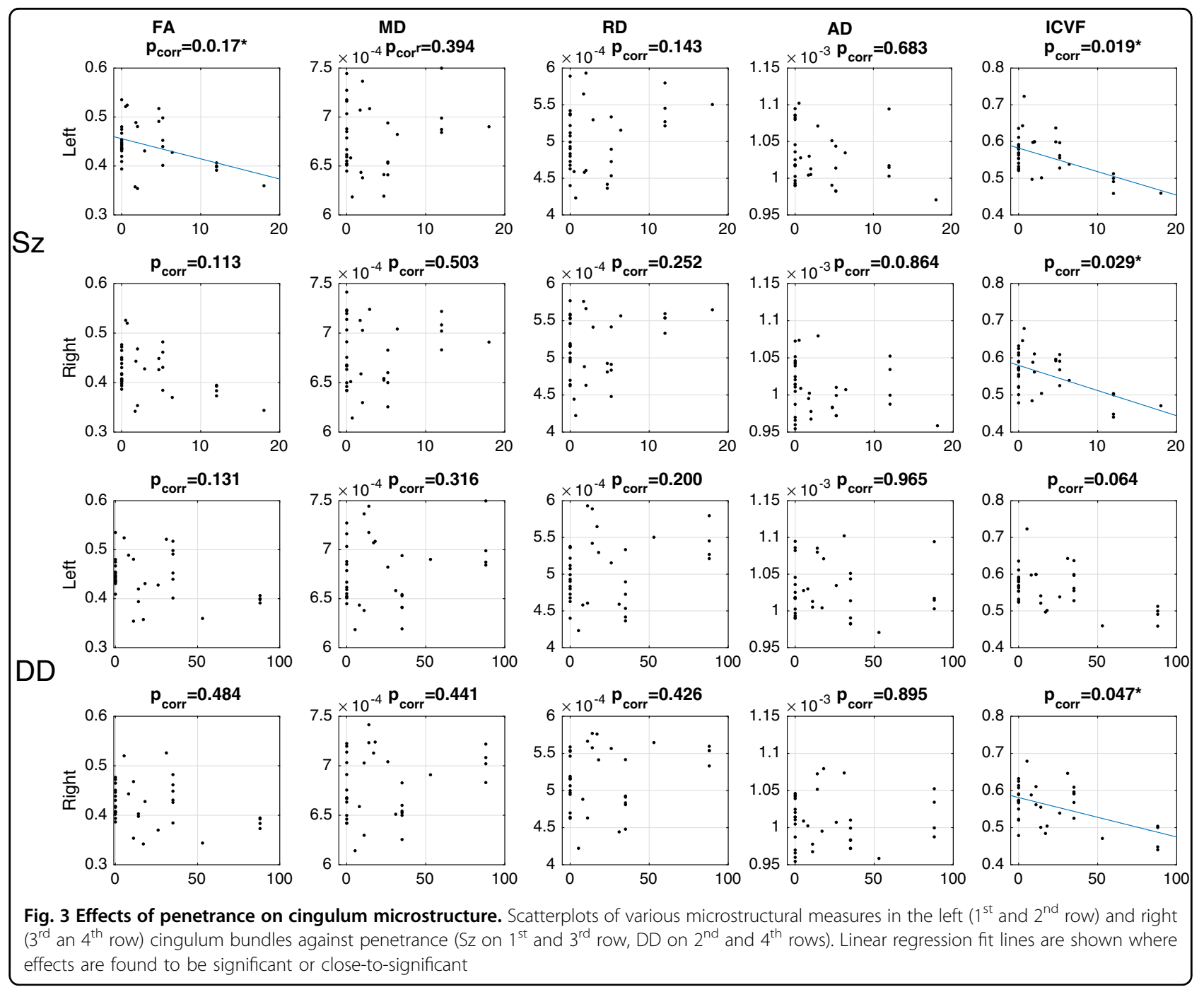

\section{Principal component analysis}

The first 34 components were tested for effects of penetrance. Only the $8^{\text {th }}$ largest component (PC8) was found to be significantly associated with $P_{\mathrm{DD}}(t=-3.033$, $\left.p=0.005, p_{\text {corr }}=0.027\right)$ and marginally significant for $P_{\mathrm{Sz}}$ $\left(t=-2.560, p=0.016, p_{\text {corr }}=0.055\right)$. This component shows no significant effect when tested with the binary CNV model $\left(t=-2.431, p=0.021, p_{\text {corr }}=0.324\right)$. This component is heavily weighted by variables relating to white-matter volume (Fig. 4). The two largest anatomical regions with the highest weighting on this component are the body and the splenium of the corpus callosum. These areas were weighted in opposite directions, which would be compatible with the altered curvature observed for the risk scores (see above, Fig. 2).

Further examination of the corpus callosum volumes, show the body is relatively smaller in high-penetrance cases (low PC8 weight) compared to low-penetrance cases (high PC8 weight). A post-hoc test was performed on the ratio of volumes between the $\mathrm{CC}$ body and splenium that confirms negative correlation with both penetrance scores $\left(P_{\mathrm{Sz}}: t=-2.193, p=0.036 ; P_{\mathrm{DD}} ; t=-2.931, p=0.006\right)$

\section{Discussion}

In this study we investigated morphological and microstructural alterations associated with CNVs with high penetrance for schizophrenia and developmental delay. We utilised a novel approach to characterising morphology of white-matter fibre bundles in combination with more traditional metrics of brain morphology and microstructure to identify features and principal components of these features that characterise neuropsychiatric CNVs. We found altered morphology in the left and right cingulum. We also found a significant reduction in FA and ICVF in this structure, and our preliminary interpretation of this result is that it reflects a reduction in 
A

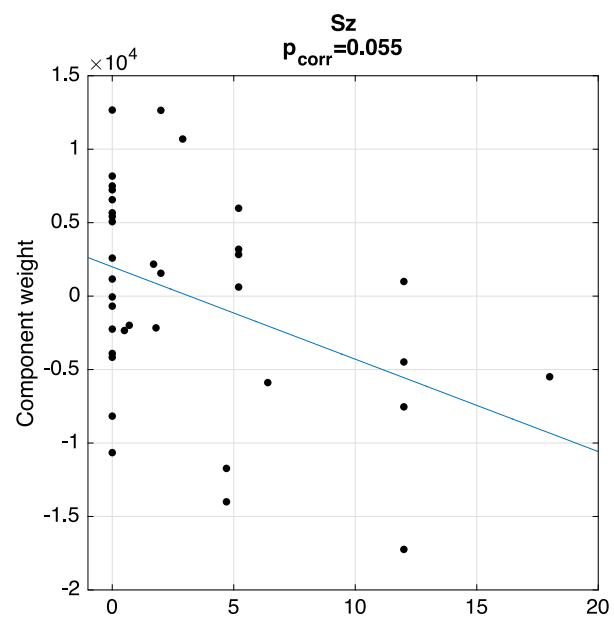

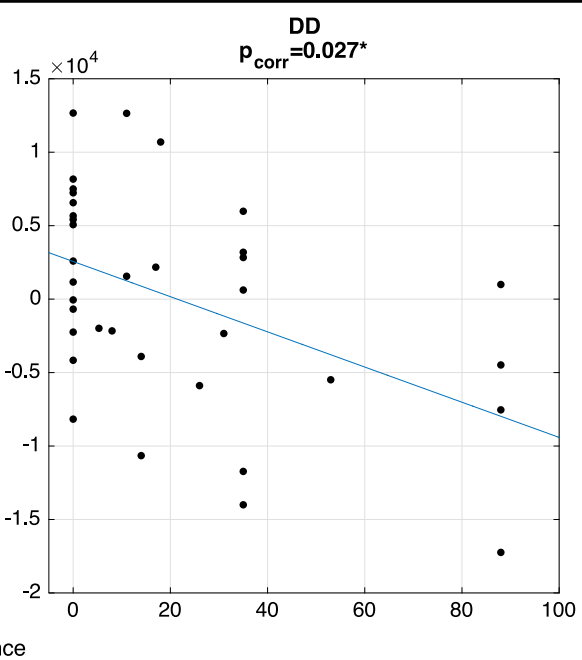

B

Penetrance WMmicro_vol_CCbody
WMmicro_vol_CCsplen WHOLEBRAIN_BrainSegVol WMmicro_vol_corticoSpinalt WMmicro_vol_CCgenu WMmicro_vol_ARCL WMmicro_vol_FORNIX_left WMmicro_vol_FORNIX_right WMmicro_vol_corticoSpinalR WHOLEBRAIN_SupraTentorialVolNotVentVox WHOLEBRAIN_SupraTentorialVoINotVent WMmicro_vol_Cing_R WHOLEBRAIN SUbCortGrayVol WHOLEBRAIN_IhCorticalWhiteMatterVol WMmicro_vol_UNCR
GM_thick_avg_rh.aparc_superiorfrontal WMmicro_vol_Cing_L GM_vol_aseg_Right-Lateral-Ventricle GM_thick_avg_lh.aparc_inferiortemporal WMmicro_vol_PHCR GM vol_aseg_Left-Cerebellum-White-Matter

GM_thick_avg_Ih.aparc_precentral WHOLEBRAIN_rhCorticalWhiteMatterVol GM_vol_wmparc_Right-UnsegmentedWhiteMatter GM_vol_wmparc_Left-UnsegmentedWhiteMatter

0

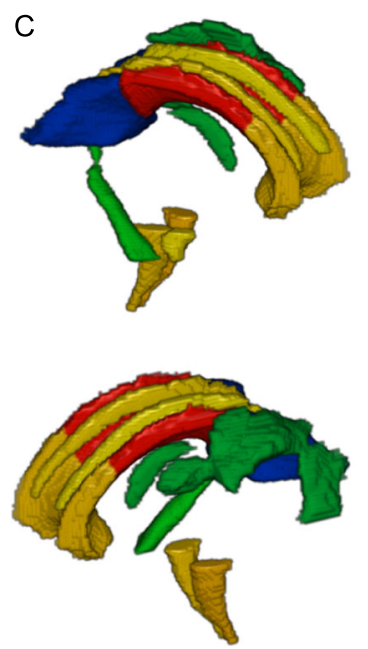

Low penetrance $\rightarrow$ Larger relative volume High penetrance $\rightarrow$ Smaller relative volume

D
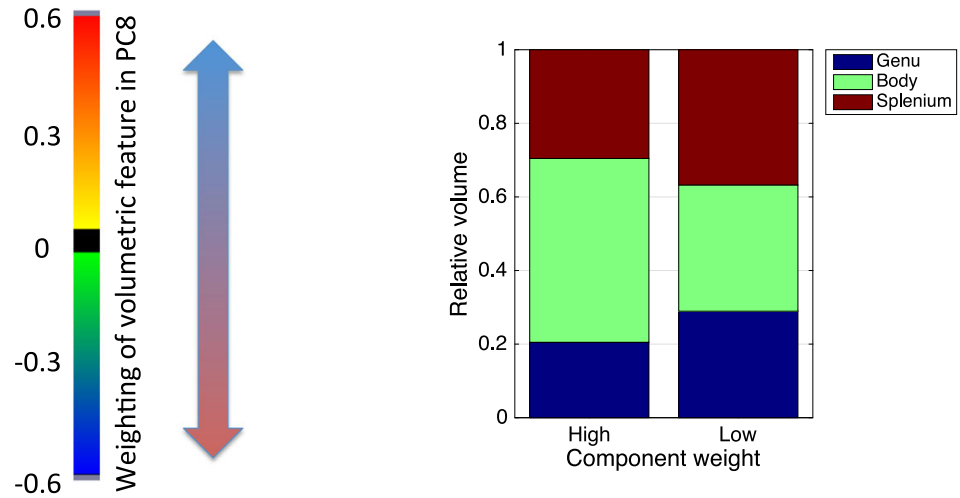

Low penetrance $\rightarrow$ Smaller relative volume High penetrance $\rightarrow$ Larger relative volume

Fig. 4 Effects of penetrance on principle component PC8. a Scatterplots of PC8 against $P_{\mathrm{Sz}}$ and $P_{\mathrm{DD}}$. $\mathbf{b}$ Weighting of each imaging variable in PC8, showing the top 25 weightings. Blue bars indicate positive weighting, red bars indicate negative weighting. $\mathbf{c}$ Volumetric change associated with white-matter structures strongly represented in PC8 rendered on the JHU atlas, with positive values corresponding to larger volumes for low penetrance (or smaller volume for higher penetrance) and negative values corresponding to smaller volumes for low penetrance (or larger volume for lower penetrance). $\mathbf{d}$ Relative volumes of 3 segments of corpus callosum for extreme cases of high and low component weight 
axon density in this pathway rather than myelin because no effects of relaxometry measures were observed (although it should be noted this may be due to comparatively lower sensitivity of R1 compared to DTI metrics $\left.{ }^{36}\right)$. There was also an overall positive trend of increased diffusivity with increased penetrance, which is also consistent with a decrease in fibre density in highpenetrance individuals.

A converging finding comes from the PCA analysis, in which PC8 shows a strong effect of penetrance. This component is heavily weighted by midline white-matter structures, in particular, the three components of the corpus callosum. Many of these volumes did not show effects when tested directly, which is likely due to discordant signs between the weighting of features within the component. Most prominently, the splenium has opposite weighting to the body and genu of the corpus callosum. This suggests that rather than there being absolute volumetric effects of penetrance, penetrance affects the volumetric interrelationships between these structures. The corpus callosum is of particular interest, because the altered interrelationships in the three segments suggest alteration of the arrangement of fibres along the AP axis. This is consistent with the increase in curvature of the cingulum along the AP axis, which wraps dorsally around the corpus callosum. We obtained these findings in the absence of any effects of gross brain morphology. There is no apparent relationship between the overall brain size or shape and penetrance. Therefore, it appears that these high-risk CNVs lead to neurodevelopmental alterations that are associated with penetrance, but manifest more subtly than in gross brain morphology. Also of note is that the results of the binary model show weak effects for these metrics, adding further credence to the view that these features are related to $\mathrm{CNV}$ penetrance for neuropsychiatric illness rather than simply due to the presence of CNVs.

Altered brain development appears to manifest in the following way: there is increase in forces ${ }^{37}$ applied parallel to the AP axis. The distribution of axons along the length of the corpus callosum is altered, which causes a change in the forces applied to the cingulum during development. Some findings from 22q11.2 deletion patients appear to corroborate this suggestion, reporting a larger area of the mid-sagittal section of the corpus callosum, in particular the posterior part ${ }^{37-39}$ which corroborates the pattern of fractional corpus callosum volumes observed in this study.

In terms of the mechanisms underlying the cingulum shape/microstructural abnormalities, the embryological literature indicates that the genes affected by the CNVs we have studied are expressed in the developing brain (e.g. for those in 22q11.2 deletion $\operatorname{see}^{40}$ ). It is therefore likely that alterations in brain morphology will start to occur at this very early stage of development, through direct effects on neurodevelopment or as secondary knock-on effects from abnormal development of the skull or interaction with environmental factors. Abnormal brain morphology can impact on mechanical processes during embryonic brain development, leading to altered structure of the cingulum and consequently morphological differences in the arrangement of fibres along the corpus callosum, as suggested by mechanical models of morphogenesis ${ }^{41}$. The early formation of neurons has been shown to guide the trajectory of axonal process ${ }^{42}$. Therefore, we can speculate that early disturbances of head shape can lead to further disruption of axonal processes, causing a reduction in axon density. There is evidence for altered neuronal migration during brain development in both humans and mouse models of 22q11.2 deletion ${ }^{43}$. Kates et al. even speculate that 22q11.2 deletion is a disorder of axonal guidance as indicated by differential developmental trajectories between 22q11.2 deletion carriers and controls in microstructural measurements ${ }^{44,45}$.

The changes in cingulum microstructure that we report are largely compatible with previous reports in schizophrenia $^{46-48}$. Similar findings have been observed in $1^{\text {st }}$ degree relatives of schizophrenia patients ${ }^{49}$ and (for right cingulum) for carriers of the common schizophrenia risk variant at ZNF840A $\mathrm{A}^{50}$ and in a sample of adolescents and young adults with 22q11.2 deletion syndrome ${ }^{51}$, where the most robust finding was AD reduction (without corresponding increases in $\mathrm{RD}$ ) in the anterior and dorsal cingulum. Although not significant, our results point to a similar trend in cingulum diffusivity measures.

Of note, these cingulum changes are observed in probands who are not affected by schizophrenia, although they do have a range of other psychiatric diagnoses (see Table S1 in SI). This suggests a putative common neurodevelopmental pathway, which is disrupted by a range of genetic variants through downstream effects on genes involved in prenatal brain development and has pleiotropic clinical effects. These features can be regarded as a hallmark of genetic vulnerability to schizophrenia, rather than of schizophrenia per se. We would also suggest that our sample, with its high rates of psychopathology and below-average IQ (see table S1 in SI), would show features of vulnerability rather than resilience. Ultimately a definitive answer to the question of mechanisms of resilience would require a much larger sample with a wider spread of levels of functioning and a larger number of highly functioning carriers without any psychopathology.

There is little discrimination between the effects of the two penetrance scores. All significant effects observed apply to both scores, with a few exceptions (for example, increased fibre dispersion in bilateral inferior frontooccipital cortices associated with higher penetrance for schizophrenia). This is also evident in the high correlation between penetrance scores. This suggests that the 
neurodevelopmental indicators observed are not attributable to a particular psychopathology, but rather that these features reflect penetrance for neuropsychiatric illness more generally, a notion which has also been proposed for the clinical phenotype ${ }^{65}$. However, it should be stressed, due to the comparatively weak effects seen in the binary model, this effect is not simply due to the presence of a CNV.

One concern of the present study the small sample size. This is an unavoidable issue in deep phenotyping research of rare genetic variations. Thus, generalisability of the findings of the present study is somewhat limited, and needs to be confirmed by replication in future studies, ideally through multi-centre collaborations. We do however mitigate issues by taking advantage of the large effect sizes in these patients and by considering variation across a spread of CNVs rather than focusing on single variants. We also need to be conscious of drawbacks of this approach. One is that contributions of individual CNVs cannot be disentangled easily, and the effects observed can be mostly driven by the contribution of a small number of very high-penetrance CNVs (see supplementary analysis in SI, section 3). It should be noted that the highly heterogeneous findings in the literature (summarised in Table 1) suggest that this type of grouped analysis risks overlooking interesting $\mathrm{CNV}$-specific mechanisms.

As noted previously, no effects of brain volume were observed, although previous CNV-specific studies have identified increased ${ }^{55,65}$ or decreased ${ }^{61-63}$ brain volume. The changes in brain volume appear to reflect CNVspecific features, but ones not necessarily related to risk for schizophrenia or developmental disorders. In contrast, our findings relating to medial white-matter structures implicate a risk mechanism for these disorders that is common across a range of high-risk genetic variants.

In summary, we reveal significant morphological and microstructural features associated with penetrance for neuropsychiatric illness in a cohort of CNV carriers. The most pronounced of these features is in the medial whitematter structures: curvature of the cingulum bundle and volumetric interrelationships between difference segments of the corpus callosum. These features are consistent with a common neurodevelopmental trajectory, which does not manifest in gross brain morphological changes, but in more subtle alterations in the morphological interrelationships in midline white-matter structures. This can lead to downstream effects on cognitive and intellectual impairments that commonly arise as a result of these genetic variants.

\section{Acknowledgements}

This study was funded by two Wellcome Trust Strategic Awards (100202/Z/12/ Z, 104943/Z/14/Z) and a Wellcome Trust New Investigator awarded to DKJ (096646/Z/11/Z). We would also like to thank the members of the field team who assisted in recruitment, assessment and data collection, Ffion Evans, Kali Barawi and Vera Schroeter.

\section{Author details}

${ }^{1}$ Cardiff University Brain Research Imaging Centre (CUBRIC), School of Psychology, Cardiff University, Maindy Road, Cardiff CF24 4HQ, United Kingdom. ${ }^{2}$ Neuroscience and Mental Health Research Institute (NMHRI), Cardiff University, Maindy Road, Cardiff CF24 4HQ, United Kingdom. ${ }^{3}$ Experimental MRI Centre (EMRIC), School of Biosciences, Cardiff University, Sir Martin Evans Building, Museum Avenue, CF10 3AX Cardiff, United Kingdom. ${ }^{4}$ MRC Centre for Neuropsychiatric Genetics and Genomics, School of Medicine, Cardiff University, Maindy Road, Cardiff CF24 4HQ, United Kingdom. ${ }^{5}$ School of Psychology, Faculty of Health Sciences, Australian Catholic University, Melbourne, VIC 3065, Australia. '5 School of Mental Health and Neuroscience, Faculty of Health, Medicine and Life Sciences, Maastricht University, Maastricht, The Netherlands

\section{Conflict of interest}

The authors declare that they have no conflict of interest.

\section{Publisher's note}

Springer Nature remains neutral with regard to jurisdictional claims in published maps and institutional affiliations.

Supplementary Information accompanies this paper at (https://doi.org/ 10.1038/s41398-019-0440-7).

Received: 28 January 2019 Accepted: 13 February 2019

Published online: 25 February 2019

\section{References}

1. Mowry, B. J. \& Gratten, J. The emerging spectrum of allelic variation in schizophrenia: current evidence and strategies for the identification and functional characterization of common and rare variants. Mol. Psychiatry 18, 38-52 (2013).

2. Owen, M. J., O'Donovan, M. C., Thapar, A. \& Craddock, N. Neurodevelopmental hypothesis of schizophrenia. Br J Psychiatry 198, 173-175 (2011).

3. Lancaster T. M. et al. Structural and Functional Neuroimaging of Polygenic Risk for Schizophrenia: A Recall-by-Genotype-Based Approach. Schizophr Bull 2018. https://doi.org/10.1093/schbul/sby037.

4. Stefansson, $H$. et al. CNVs conferring risk of autism or schizophrenia affect cognition in controls. Nature 505, 361-366 (2014).

5. Boot, E. \& van Amelsvoort, T. A. M. J. Neuroimaging correlates of $22 q 11.2$ deletion syndrome: implications for schizophrenia research. Curr. Top. Med. Chem. 12, 2303-2313 (2012).

6. Zinkstok, J. \& van Amelsvoort, T. Neuropsychological profile and neuroimaging in patients with 22Q11.2 deletion syndrome: a review keywords. Child Neuropsychol. 11, 21-37 (2005).

7. Tan, G. M., Arnone, D., Mclntosh, A. M. \& Ebmeier, K. P. Meta-analysis of magnetic resonance imaging studies in chromosome $22 q 11.2$ deletion syndrome (velocardiofacial syndrome). Schizophr. Res. 115, 173-181 (2009).

8. Sun D. et al. Large-scale mapping of cortical alterations in 22q11.2 deletion syndrome: convergence with idiopathic psychosis and effects of deletion size. Mol Psychiatry 2018. https://doi.org/10.1038/s41380-018-0078-5.

9. Ulfarsson, M. O. et al. 15q11.2 CNV affects cognitive, structural and functional correlates of dyslexia and dyscalculia. Transl. Psychiatry 7, e1109 (2017).

10. Kirov, G. et al. The penetrance of copy number variations for schizophrenia and developmental delay. Biol. Psychiatry 75, 378-385 (2014).

11. Parker G. D., Evans G. J. A., Jones D. K. A novel framework for global comparison of tract-topology between subjects reveals callosum shape variations in first episode psychosis. In Proc. of the International Society for Magnetic Resonance in Medicine. p. 4131. (ISMRM, Singapore, 2016).

12. Zhang, H. Schneider, T., Wheeler-Kingshott, C. A. \& Alexander, D. C. NODDI: practical in vivo neurite orientation dispersion and density imaging of the human brain. Neuroimage 61, 1000-1016 (2012).

13. Deoni, S. C. L. High-resolution $T 1$ mapping of the brain at $3 T$ with driven equilibrium single pulse observation of $\mathrm{T} 1$ with high-speed incorporation of 
RF field inhomogeneities (DESPOT1-HFI). J. Magn. Reson. Imaging 26, 1106-1111 (2007).

14. Wang, K. et al. PennCNV: an integrated hidden Markov model designed for high-resolution copy number variation detection in whole-genome SNP genotyping data. Genome Res. 17, 1665-1674 (2007).

15. Jones, D. K., Simmons, A., Williams, S. C. R. \& Horsfield, M. A. Non-invasive assessment of axonal fiber connectivity in the human brain via diffusion tensor MRI. Magn. Reson. Med. 42, 37-41 (1999).

16. Fischl, B. FreeSurfer. Neuroimage 62, 774-781 (2012).

17. Dale, A. M., Fischl, B. \& Sereno, M. I. Cortical surface-based analysis. I. Segmentation and surface reconstruction. Neuroimage 9, 179-194 (1999).

18. Ségonne, F. et al. A hybrid approach to the skull stripping problem in MRI. Neuroimage 22, 1060-1075 (2004).

19. Fischl B., et al. Sequence-independent segmentation of magnetic resonance images. Neurolmage. 2004 https://doi.org/10.1016/j.neuroimage.2004.07.016.

20. Fischl, B., Liu, A. \& Dale, A. M. Automated manifold surgery: constructing geometrically accurate and topologically correct models of the human cerebral cortex. IEEE Trans. Med. Imaging 20, 70-80 (2001).

21. Fischl, B., Sereno, M. I., Tootell, R. B. H. \& Dale, A. M. High-resolution intersubject averaging and a coordinate system for the cortical surface. Hum. Brain. Mapp. 8, 272-284 (1999)

22. Fischl, B., Sereno, M. I. \& Dale, A. M. Cortical surface-based analysis: II. Inflation, flattening, and a surface-based coordinate system. Neuroimage 9, 195-207 (1999).

23. Reuter, M., Schmansky, N. J., Rosas, H. D. \& Fischl, B. Within-subject template estimation for unbiased longitudinal image analysis. Neuroimage $\mathbf{6 1}$ 1402-1418 (2012).

24. Desikan, R. S. et al. An automated labeling system for subdividing the human cerebral cortex on MRI scans into gyral based regions of interest. Neuroimage 31, 968-980 (2006).

25. Jenkinson, M., Beckmann, C. F., Behrens, T. E. J., Woolrich, M. W. \& Smith, S. M. FSL. Neuroimage 62, 782-790 (2012).

26. Smith, S. M. Fast robust automated brain extraction. Hum. Brain. Mapp. 17, 143-155 (2002).

27. Leemans A., Jeurissen B., Sijbers J., Jones D. K. ExploreDTI: a graphical toolbox for processing, analyzing, and visualizing diffusion MR data. In Proc. of the International Society for Magnetic Resonance in Medicine. 3537 (ISMRM, Honolulu, Hawai'i, USA, 2009).

28. Leemans, A. \& Jones, D. K. The B-matrix must be rotated when correcting for subject motion in DTI data. Magn. Reson. Med. 61, 1336-1349 (2009).

29. Wu, M., Chang, L. C., Walker, L., Lemaitre, H., Barnett, aS. \& Marenco, S. et al. Comparison of EPI distortion correction methods in diffusion tensor MRI using a novel framework. Med Image Comput. Comput. Interv 11, 321-329 (2008).

30. Klein, S., Staring, M., Murphy, K, Viergever, Ma \& Pluim, J. P. W. Elastix: a toolbox for intensity-based medical image registration. IEEE Trans. Med. Imaging 29, 196-205 (2010).

31. Pasternak, O., Sochen, N., Gur, Y., Intrator, N. \& Assaf, Y. Free water elimination and mapping from diffusion MRI. Magn. Reson. Med. 62, 717-730 (2009).

32. Dell'acqua, F. et al. A modified damped Richardson-Lucy algorithm to reduce isotropic background effects in spherical deconvolution. Neuroimage 49, 1446-1458 (2010).

33. Tournier, J.-D., Calamante, F. \& Connelly, A. Robust determination of the fibre orientation distribution in diffusion MRl: non-negativity constrained superresolved spherical deconvolution. Neuroimage 35, 1459-1472 (2007).

34. Parker G. D., et al RESDORE: Robust Estimation in Spherical Deconvolution by Outlier Rejection. In: Proc of the International Society for Magnetic Resonance in Medicine. p. 3148 (ISMRM, Salt Lake City, USA., 2013).

35. Basser, P. J., Pajevic, S., Pierpaoli, C., Duda, J. \& Aldroubi, A. In vivo fiber tractography using DT-MRI data. Magn. Reson. Med. 44, 625-632 (2000).

36. De Santis, S., Drakesmith, M., Bells, S., Assaf, Y. \& Jones, D. K. Why diffusion tensor MRI does well only some of the time: variance and covariance of white matter tissue microstructure attributes in the living human brain. Neuroimage 89, 35-44 (2014).

37. Shashi, V. et al. Abnormalities of the corpus callosum in nonpsychotic children with chromosome 22q11 deletion syndrome. Neuroimage 21, 1399-1406 (2004).

38. Antshel, K. M., Conchelos, J., Lanzetta, G., Fremont, W. \& Kates, W. R. Behavior and corpus callosum morphology relationships in velocardiofacial syndrome (22q11.2 deletion syndrome). Psychiatry Res Neuroimaging 138, 235-245 (2005).

39. Shashi, V. et al. Increased corpus callosum volume in children with chromosome 22q11.2 deletion syndrome is associated with neurocognitive deficits and genetic polymorphisms. Eur. J. Hum. Genet. 20, 1051-1057 (2012).

40. Maynard, T. M. et al. A comprehensive analysis of 22q11 gene expression in the developing and adult brain. Proc. Natl Acad. Sci. USA 100, 14433-14438 (2003).

41. Van Essen, D. C. A tension-based theory of morphogenesis and compact wiring in the central nervous system. Nature 385, 313-318 (1997).

42. López-Bendito, G. et al. Tangential neuronal migration controls axon guidance: a role for Neuregulin-1 in thalamocortical axon navigation. Cell 125, 127-142 (2006).

43. Meechan, D. W. et al. Modeling a model: mouse genetics, 22q11.2 Deletion Syndrome, and disorders of cortical circuit development. Prog. Neurobiol. 130, $1-28$ (2015)

44. Kikinis, Z. et al. Reduced fractional anisotropy and axial diffusivity in white matter in 22q11.2 deletion syndrome: a pilot study. Schizophr. Res. 141, 35-39 (2012).

45. Kikinis, Z. et al. Genetic contributions to changes of fiber tracts of ventral visual stream in 22q11.2 deletion syndrome. Brain. Imaging Behav. 7, 316-325 (2013).

46. Whitford, T. J. et al. Localized abnormalities in the cingulum bundle in patients with schizophrenia: a diffusion tensor tractography study. Neuroimage Clin. $\mathbf{5}$ 93-99 (2014).

47. Abdul-Rahman, M. F., Qiu, A. \& Sim, K. Regionally specific white matter disruptions of fornix and cingulum in schizophrenia. PLOS ONE 6, e18652 (2011).

48. Wheeler, A. L. \& Voineskos, A. N. A review of structural neuroimaging in schizophrenia: from connectivity to connectomics. Front. Hum. Neurosci. 8 1-18 (2014).

49. Knöchel, C. et al. Association between white matter fiber integrity and subclinical psychotic symptoms in schizophrenia patients and unaffected relatives. Schizophr. Res. 140, 129-135 (2012).

50. Mallas, E.-J. et al. Genome-wide discovered psychosis-risk gene ZNF804A impacts on white matter microstructure in health, schizophrenia and bipolar disorder. PeerJ 4, e1570 (2016).

51. Kates, W. R. et al. White matter microstructural abnormalities of the cingulum bundle in youths with 22q11.2 deletion syndrome: Associations with medication, neuropsychological function, and prodromal symptoms of psychosis. Schizophr. Res. 161, 76-84 (2015).

52. Boronat, S., Mehan, W. A., Shaaya, E. A., Thibert, R. L. \& Caruso, P. Hippocampa abnormalities in magnetic resonance imaging (MRI) of $15 q$ duplication syndromes. J. Child Neurol. 30, 333-338 (2015).

53. Beal, J. C. Case Report: neuronal migration disorder associated with chromosome 15q13.3 duplication in a boy with autism and seizures. J. Child Neurol. 29, NP186-NP188 (2014).

54. Barber, J. C. K. et al. 16p11.2-p12.2 duplication syndrome; a genomic condition differentiated from euchromatic variation of 16p11.2. Eur. J. Hum. Genet. 21, 182-189 (2013).

55. Qureshi, A. Y. et al. Opposing brain differences in 16p11.2 deletion and duplication carriers. J. Neurosci. 34, 11199-11211 (2014).

56. Maillard, A. M. et al. The 16p11.2 locus modulates brain structures common to autism, schizophrenia and obesity. Mol. Psychiatry 20, 140-147 (2015).

57. Martin-Brevet, S. et al. Quantifying the effects of 16p11.2 Copy number variants on brain structure: a multisite genetic-first study. Biol. Psychiatry 84, 253-264 (2018).

58. Owen, J. P. et al. Aberrant white matter microstructure in children with 16p11.2 deletions. J. Neurosci. 34, 6214-6223 (2014).

59. Carbunar, O. \& Bicknese, A. MRI Findings with duplication of $17 q 12$ Chromosome region in a 17 year old (P02.181). Neurology 78(P02), 181 (2012).

60. Bernier, R. et al. Clinical phenotype of the recurrent 1q21.1 copy-number variant. Genet. Med. 18, 341-349 (2016).

61. Kates, W. R. et al. Regional cortical white matter reductions in velocardiofacial syndrome: a volumetric MRI analysis. Biol. Psychiatry 49, 677-684 (2001).

62. Eliez, S., Schmitt, J. E., White, C. D. \& Reiss, A. L. Children and adolescents with velocardiofacial syndrome: a volumetric MRI Study. Am. J. Psychiatry 157, 409-415 (2000). 
63. Bearden, C. E. et al. Mapping cortical thickness in children with $22 q 11.2$ deletions. Cereb. Cortex 17, 1889-1898 (2007).

64. Jalbrzikowski, M. et al. Structural abnormalities in cortical volume, thickness, and surface area in 22q11.2 microdeletion syndrome: Relationship with psychotic symptoms. Neuroimage Clin. 3, 405-415 (2013).

65. Olszewski, A. K. et al. The social brain network in 22q11.2 deletion syndrome: a diffusion tensor imaging study. Behav. Brain Funct. 13, 4 (2017).

66. Schmitt, J. E. et al. Aberrant cortical morphometry in the 22q11.2 deletion syndrome. Biol. Psychiatry 78, 135-143 (2015).
67. Roalf, D. R. et al. White matter microstructural deficits in 22q11.2 deletion syndrome. Psychiatry Res. Neuroimaging 268, 35-44 (2017).

68. Ching, M. S. et al. Deletions of NRXN1 (neurexin-1) predispose to a wide spectrum of developmental disorders. Am. J. Med. Genet. B Neuropsychiatr. Genet. 153B, 937-947 (2010).

69. Duong, L. et al. Mutations in NRXN1 in a family multiply affected with brain disorders: NRXN1 mutations and brain disorders. Am. J. Med Genet. B Neuropsychiatr. Genet. 159B, 354-358 (2012). 